\title{
International trade policies and sustainability of nano-textiles
}

\author{
Badri G. Narayanan
}

Economist, School of Environment and Forestry Sciences, University of Washington Seattle, USA

Naveen R. Kadasala

Postdoctoral Research Associate, Faculty of Material Science Engineering, Purdue University, USA

\section{L.V. Narayan Achari}

Postdoctoral Research Associate, Faculty of Material Science Engineering, Purdue University, USA

\section{Seshadev Sahoo}

Postdoctoral Research Associate, Faculty of Material Science Engineering, Purdue University, USA

\begin{abstract}
While a considerable part of developments in the field of industrial and technical textiles involves nanomaterials, there are increasing challenges faced across the world in their acceptability and sustainability. Their potential adverse impact has been documented in the scientific literature. European Commission and USEPA (United States Environmental Protection Agency) have already come up with regulations that govern these materials. In future, therefore, we can expect trade sanctions by these countries on exports of nano-textiles from countries like India. In this paper, we shall discuss the technical literature that points towards potential hazards of nano-textiles and the policy aspects currently in place. Further, we employ a widely used global economic (Computable General Equilibrium) model (GTAP - Global Trade Analysis Project) to evaluate the economic and business impact of such sanctions quantitatively, in a stochastic and comparative setting.
\end{abstract}

Keywords: international trade, environment, health, BPA, plastics.

JEL Classification: F13, F18, C68, I18, L5, N4.

DOI: $10.21272 / \mathrm{sec} .1(2) .27-33.2017$.

(C) The Authors, 2017. This article is published with open access at ARMG Publishing.

\section{Introduction}

Nanotechnology and nano-materials enabled by it are rapidly expanding all over the world. Studies predict a global market to the tune of \$1.5-3 trillion US\$ in this area. Several companies are involved in the research, development and manufacture of nano-materials [1]. In USA, there are about 900 companies focusing on this, while in each of Germany, UK and Canada, there over 100 of them. China has close to 50 of them, while India has about a dozen; however, the industry is growing rapidly in India and China as well. Nanomaterials and technologies are widely used in textiles increasingly [2-4]. However, there have been some concerns on their sustainability since the recent past [5-8].

In this study, we examine the scenarios in the future, wherein the nano-technologies and nano-materials are likely to be more regulated and restricted across the world, in terms of production and trade. We focus on US and EU as the major policy influencers, based on the current developments, and assume market access restrictions as well as control of production of these materials. We analyze the impact of such uncertain policy shifts on the textile industries in countries like India and China, employing the widely used global simulation model: Global Trade Analysis Project (GTAP). This paper is organized as follows: section 2 discusses the literature, section 3 provides the methodology, section 4 discusses the results and section 5 concludes.

\section{Literature review}

While the benefits of nano-technology are widely known and acknowledged for the decades now, there has been new literature on toxicity and health effects of nano-materials. Many studies such as Peterson et al. (2007) and Handy and Shaw (2007) indicate that the channels through which nano-particles can affect the 
environment and health are unclear, although their toxicity is not as uncertain [8-11]. The debate here is whether they affect the workers due to exposure to nano-particles or they also affect the people at large through the consumption of new products made of them [12]. The evidence is more for the former than for the latter, but research is ongoing in this area. To the extent that the evidence is strong on the toxicity levels of nano-particles, the policies adopted by countries, particularly the developed world, will be stringent against them [13].

Nevertheless, the public perception matters a lot and this is what influences policy in the democratic world devoid of undue lobbying pressures. Nerliche, Clarke and Ulph (2007) provides evidence in the UK that the public perception is not much in favor of nano-particles, indicating that the regulations governing them are likely to be much more stringent in the future than it is today [14]. Powell (2007) concludes that the scientists involved in nanotechnology development overwhelmingly believe that nano-particles and technologies offer no new risks, while the environmental and health scientists who work on the toxicity effects of these materials have a consensus that they offer significant and new risks, based on a detailed survey among scientists [15]. In future, policy-making will depend on joint participation of these groups of scientists.

Based on these studies, it is apparent that the policy-makers are going to be less sympathetic of nanoparticles in the future. This is also evident from the policy reports of the developed countries. The following is text quoted from European Commission's study conducted by Ganzleben et al. (2011): "Releases during product use will depend upon the mobility of the nanomaterials within the product, in particular where they are relatively immobile within a solid matrix, more easily dispersed within a liquid or even set for intentional release. It is expected that release will be most significant from creams and cosmetics that contain nanomaterials and that are washed off into sewage systems following use, as well as from washing textiles that contain nanomaterials and detergents and washing machines that use nanosilver. In addition, spillages of oils, fuels and lubricants containing nanomaterials provide a likely exposure pathway onto roads, with wash-off into sewage systems, soil and surface waters likely. Releases of nanomaterials from solid matrices through wear and tear are considered less significant in terms of volume. In addition, there are some applications of nanomaterials where their release into the environment is inherent to their function soil remediation, water purification, cleaning etc.

Sewage waters will then be treated in urban waste water treatment plants, with nanomaterials either collecting in sewage sludge or passing through the treatment processes and out into surface waters with the release of treated effluent. Sewage sludge spread on agricultural land may then contaminate soils with nanomaterials.

With regards to the disposal phase of the product life cycle, the likelihood of environmental exposure will be influenced by the mobility of the nanomaterials in the waste product and the waste treatment process applied. Incineration may result in emissions of nanomaterials to air and ashes containing nanomaterials that have not been degraded (such as carbon nanotubes). There is also the possibility of nanomaterials entering landfill passing into leachate and entering groundwater, particularly in older landfill sites that may not be lined.

These potential pathways are all subject to control under EU legislation, most of which are addressed in this report with the exception of industrial emissions which are addressed in a related study. The exposure pathways and the coverage provided by relevant legislation are discussed in greater detail under the sections on relevant legislation.

With regard to releases of nanomaterials over the product life cycle, it seems important to distinguish between applications that are inherently dispersive (e.g. nanomaterials in liquids) and applications that are not inherently dispersive (e.g. nanomaterials in a solid matrix).

Once nanomaterials have entered the environment, the associated degree of risk will depend upon the mobility of the nanomaterials in the parent matrix as well as potential hazard characteristics, as shown in box 2 below. When considering the stringency of possible controls, the dispersive nature of the nanomaterials should feed into the analysis [16]."

Duvall and Wyatt (2011) mention that similar stringent policies are under consideration in the US as well. Below is a quote from this study: "In the United States, the Environmental Protection Agency (EPA) is shifting from a voluntary to a mandatory approach to regulating and collecting information on the potentially novel risks from nanomaterials. Under the Toxic Substances Control Act (TSCA), in particular, EPA has reviewed more than 100 premanufacture notices for nanomaterials and has imposed restrictions on many of 
them. EPA is taking advantage of its current significant new use rule (SNUR) authority to impose restrictions and gather data on nanomaterials, with several SNURs for nanomaterials already on the books and a categorical SNUR for other nanomaterials in development. EPA is also developing a mandatory data submission rule and a testing rule for certain nanomaterials, and may be given more authority to regulate nanomaterials if TSCA legislation proceeds through Congress. EPA's efforts under TSCA are complemented by data gathering and regulatory measures under the Federal Insecticide, Fungicide, and Rodenticide Act, which regulates antimicrobial nanomaterials like nanosilver. A nanosilver active ingredient is in the process of potentially being conditionally registered, and EPA has also used nanosilver in a major draft case study. Other federal agencies and U.S. states and localities are also devoting attention to nanomaterials and in some cases imposing requirements.

Outside the United States, nanomaterial regulatory requirements are most prominent in the European Union, where nanomaterials are singled out for regulation under the Cosmetics Directive and where regulators are considering future alterations to the REACH Regulation and to the Restriction of Hazardous Substances (RoHS) Directive related to nanomaterials. Many other countries have also taken or are considering actions to regulate nanomaterials. Activities by other international agencies and organizations may have significant impacts on nanomaterial producers, processors, and users [17]."

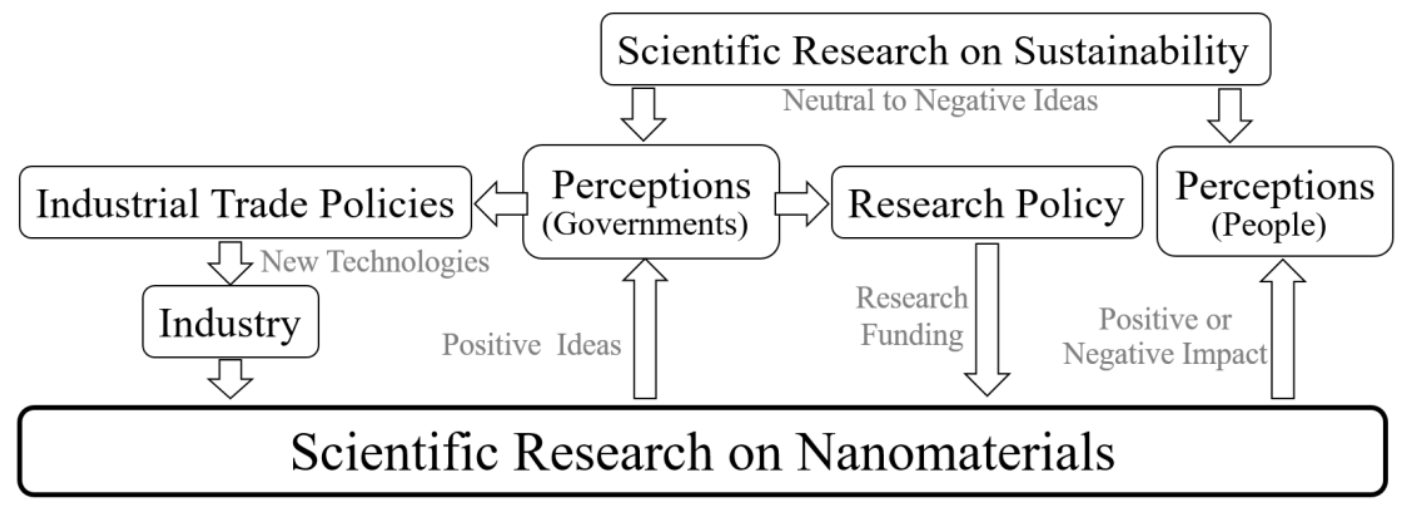

Figure 1. Overview of policy mechanisms affecting the nano-industry

We attempt to provide an overview of the policy mechanisms involved in the regulation of nano-materials, in Figure 1, based on the literature review done herein. We observe a divergence of views between scientists working on developing new nano-materials and those concerned about sustainability and public health issues related to them. While the former have focused solely on technology development and at best, a rebuttal of concerns raised by the latter, the emphasis of the latter has been on negative effects of these materials or at best, neutral ideas on their sustainability impact. All these ideas are fed into the public at large as well as to the governments and policy makers directly. People and policy-makers form perceptions based on these ideas. These perceptions are not often based on thorough reading of the literature as well as practical aspects involved, but the popular media coverage of scientific thoughts may play a key role in such transmission of ideas.

People's perceptions affect those of the governments in most of the democratic governments today, despite the industrial lobbies and corruption. On the other hand, the policy-makers can also influence the perceptions of people, which, in turn, can feed back into the government's thought process. The result of such feedback and interactions between the people and leaders is the policy framework comprising research and industrial aspects. Industrial policies may involve regulations on production of these nano-materials, while research policies would shape the public funding scenario of their further research and development in future.

\section{Methodology}

Several models may be employed to analyze the economic impact of policies in the future, but many of them ignore a few key aspects of the economy, such as the economy-wide constraints of factor endowments and resources and inter-sectoral linkages, which can be well-captured in a general equilibrium framework. Computable General Equilibrium (CGE) models take these aspects into consideration using extensive datasets. GTAP (Global Trade Analysis Project) model (Hertel, 1997), for example, is very generic and aggregated in terms of sectors and regions, but contains detailed linkages across countries and sectors as well as 
policy instruments [18]. Thus, it may be well-suited for analysis of global policy effects in nanotechnology sector.

In this section we discuss a few major features of the GTAP model developed by the Center for Global Trade Analysis at Purdue University. Such models may serve the best if one is interested in policy impact, particularly with an inter-sectoral linkages and constrained resources/factors perspective. GTAP Data Base is the dataset used in tandem with this model as well as several other global CGE models (Narayanan et al 2012) [19].

GTAP model is defined in linearized difference equations, therefore most of the variables are in percentage change. Each country/region is represented by a regional household, which has a Cobb-Douglas utility function that distributes aggregate demand into three different categories in every regional household, namely, savings, private households and government. Regional household's income comes from various taxes and primary factor payments. Savings from each region are accumulated into global savings, which is allocated to different regions as investment based on the movement of prices of capital goods as well as expected rate of return inferred from the capital stock in the beginning and end of the simulation period.

For a traded commodity $i$ in region $r$, market clearing is satisfied by equating percentage changes in output (qo) with share-weighted percentage changes in various demand sources of domestic output, viz, local consumption of domestic demand $(q d s)$ and exports $(q x w)$; with a slack variable that can be made endogenous to introduce market disequilibrium if needed.

This equation assures market clearing in the traded goods markets:

$q o(i, r)=\operatorname{SHRDM}(i, r) * q d s(i, r)+\operatorname{SHRXM}(i, r) * q x w(i, r)+\operatorname{tradslack}(i, r)$.

There are similar market clearing conditions for imports (by users of imports, as firms, households and government), domestic consumption (by users as assigned for imports) and endowment output (by usage in various sectors). Zero profits are assumed in the standard form of this model, implying perfect competition, although there can be imperfect competition incorporated by considering increasing returns to scale and monopolistic competition. This condition is employed to infer the endogenous output change in every sector.

Various types of prices in the model are linked with each other through tax/subsidy wedges, which exist across the user types, output and source of use and production. Armington assumption helps differentiate domestic commodities from imports as well as imports from one source with those from another. Trade links the regions, each of which has an identical model structure being outlined herein. The percentage change in imports of commodity $i$ from region $r$ to region $s$ (qxs), for example, is derived from two terms: expansion term that arises from the overall change in aggregate imports in region $s$ and substitution term that captures the shift of demand from one source to another, based on the Armington elasticity and the difference between percentage changes in bilateral import prices (pms) and those in the destination-generic aggregate prices ( $\mathrm{pim}$ ). An additional, typically exogenous, variable ams captures the technological change augmented by a particular bilateral trade flow:

$q x s(i, r, s)=\operatorname{qim}(i, s)+\operatorname{ams}(i, r, s)-\operatorname{ARMINGTON}(i) *\{p m s(i, r, s)-\operatorname{pim}(i, s)+\operatorname{ams}(i, r, s)\}$.

Demand for commodities across the user types has a two-stage process: first, the user $(q f, q p$ and $q g$ for firms, private household and government, respectively) decides the total demand, based on the regional household's utility function; secondly, each user decides how much of it needs to come from domestic $\mathrm{d}$ and from imports $m$. For firms, for example, the change in domestic consumption of commodity $i$ used in the production of commodity $j$ in region $r$ is simulated by the overall change (domestic + imports) in this particular consumption (expansion effect) and the domestic-import Armington elasticity multiplied by the differential between domestic prices of commodity $i$ used to produce commodity $j$ and aggregated (weighted average of domestic and imported prices) prices of the same, as shown below:

$$
\begin{aligned}
q f d(i, j, s) & =q f(i, j, s) \\
- & \text { ARMINGTON_D(i) } *[p f d(i, j, s)-p f(i, j, s)]
\end{aligned}
$$

The counterpart for import demand for the equation above is as follows:

$q f m(i, j, s)=q f(i, j, s)$

$$
\text { - ARMINGTON_D(i)*[pfm(i,j,s) - pf(i,j,s)]; }
$$


Private households determine their expenditure based on a per capita Constant Difference Elasticities (CDE) implicit expenditure function. Production is depicted in a multi-nest system. On the top, firms decide to produce certain quantities of output; a Leontief structure is then used to choose between the value-added composite commodity and intermediate input composite commodity; CES nests are then defined among the value-added categories (usage of various factors) and different intermediate inputs. Welfare of different regions is represented by Equivalent Variations (EV). This may be decomposed into many different components from the model and various aspects such as allocative efficiency, terms of trade, technology, endowment effect, investment-savings adjustment, etc.

With such a model structure, we undertake one simulation: reduction of all manufacturing sectors by a small fraction, which is calculated as the contribution of nano-sector in the global economic output. This varies between 2 and $4 \%$ for many countries in the world; for China and India, it is $2 \%$, while for EU, USA and Canada, it is $4 \%$. Since it is not certain as to what will be the actual extent of reduction, we purse a systematic sensitivity analysis (Malcolm, 1998), which involves varying the shocks between $0 \%$ and $200 \%$ of the actual shocks, thereby introducing stochastics into the system. Thus, in effect, we vary the shocks for China and India between $0 \%$ and $4 \%$ and those for USA, EU and Canada between $0 \%$ and $8 \%$.

\section{Results}

Overall, the results suggest a visible economic impact of regulations on nano-materials and nanotechnology. Table 1 summarizes the simulation results for GDP and national income for selected countries. The results suggest that while there is a risk of huge reduction in GDP and income in a pessimistic scenario, an optimistic one suggests positive changes in both of them for all countries. China is the biggest potential loser, since it also depends on US and EU-27 a lot, through the trade linkages, thereby doubly affected by domestic output reduction and global reduction. India also has the potential to lose as much as US, Canada and EU. The numbers shown here - 13-40\% reduction in GDP and income, is quite significant; in terms of loss in income, this translates into over 200 billion US\$ loss in Canada to over 3 trillion US\$ loss in USA. The upper bound is far less pronounced, indicating that in a real-world scenario, we would still see huge reduction in global output, mainly in China, USA and EU, if nano-materials are banned.

Table 1. GDP and national income: selected results (95\% confidence interval)

\begin{tabular}{|l|c|c|c|c|c|c|}
\hline & \multicolumn{2}{|c|}{ GDP change in percent } & \multicolumn{2}{c|}{ Income change in percent } & \multicolumn{2}{c|}{ Income change in US\$ bn } \\
\hline Region & Lower bound & Upper bound & Lower bound & Upper bound & Lower bound & Upper bound \\
\hline China & -39.4 & 11.5 & -37.2 & 10.9 & -2583.2 & 754.3 \\
\hline Bangladesh & -1.1 & 0.3 & -3.3 & 1.0 & -3.5 & 1.0 \\
\hline India & -13.8 & 4.0 & -15.9 & 4.6 & -280.4 & 81.9 \\
\hline Canada & -12.9 & 3.8 & -13.1 & 3.8 & -216.9 & 63.3 \\
\hline USA & -17.4 & 5.1 & -22.2 & 6.5 & -3104.1 & 906.4 \\
\hline Brazil & -0.1 & 0.4 & -0.3 & 1.0 & -7.1 & 24.3 \\
\hline EU27 & -16.4 & 4.8 & -18.0 & 5.3 & -2923.7 & 853.7 \\
\hline Russia & -0.4 & 0.1 & -0.2 & 0.6 & -3.2 & 10.9 \\
\hline
\end{tabular}

Table 2 focuses on global textile sector. These results show that employment losses may also be considerable in the textile sectors in all countries considered herein, as seen from the lower bound. However, unlike the overall GDP/income losses shown in Table 1, the numbers in upper bound are quite high, implying that on an average, in a real world situation, employment may not decline a lot in the textile sector. Similar story emerges for capital use in textile sector. However, textile prices may increase a lot, as seen in the huge upper bounds and relatively small negative lower bounds. This partly explains why more labor and capital may be drawn into textile sector despite decline in output - higher market prices attract more resources to enter the textile sector.

From the other results of this simulation we observe a sharp fall in domestic consumption of textiles owing to high prices, increased exports from countries like India, increased imports by countries like China and Brazil, etc. Still, India and China have positive trade balance change, on an average, at the cost of huge negative trade balance change in countries like US, Canada and EU. Huge implicit decline of productivity ranging from 4 to $17 \%$ may result from this policy. In other words, the global textile sector may get re-organized as a result of banning nano-materials. 
Table 2. Selected results (in percent changes) for global textiles sectors (95\% confidence interval)

\begin{tabular}{|l|c|c|c|c|c|c|}
\hline & \multicolumn{2}{|c|}{ Textile employment } & \multicolumn{2}{c|}{ Textile capital } & \multicolumn{2}{c|}{ Textile prices } \\
\hline Region & Lower bound & Upper bound & Lower bound & Upper bound & Lower bound & Upper bound \\
\hline China & -2.4 & 8.3 & -1.2 & 4.2 & -10.1 & 34.5 \\
\hline Bangladesh & -4.5 & 15.4 & -4.5 & 15.6 & -6.8 & 23.2 \\
\hline India & -3.9 & 13.5 & -2.9 & 9.9 & -9.2 & 31.3 \\
\hline Canada & -3.0 & 10.2 & -2.2 & 7.8 & -9.8 & 33.6 \\
\hline USA & -7.1 & 24.6 & -7.0 & 24.0 & -9.8 & 33.7 \\
\hline Brazil & -1.2 & 0.4 & -1.3 & 0.4 & -9.9 & 33.9 \\
\hline EU27 & -2.3 & 8.0 & -2.6 & 8.9 & -9.8 & 33.6 \\
\hline Russia & -15.6 & 4.6 & -16.5 & 4.8 & -10.6 & 36.2 \\
\hline
\end{tabular}

\section{Conclusions}

Visible changes in the global economy can occur if nano-materials are heavily restricted. Countries like China may lose a lot, while others like India may lose moderately, while trade balance may get further skewed towards these two countries in sectors such as textiles. On the other hand, changes in employment and capital in textile sector may be rather mild, because of the implicit decline in productivity as well as increased prices that attract resources from other sectors to textiles. On balance, it appears that extensive regulation of nano-materials may lead to adverse global consequences. However, sustainability is important, therefore the policy-makers might require deep thinking before taking any major steps in this direction. Perhaps a phased approach to enhancing standards related to sustainability of these nano-materials could help minimizing the economic losses seen in this paper.

\section{References}

1. Aitken, R., et al. (2006). Manufacture and use of nanomaterials: current status in the UK and global trends. Occupational Medicine, 56(5), 300-306.

2. Ulrich, C. (2006). Nano-textiles are engineering a safer world. Human Ecology, 34(2), 2.

3. Mitrano, D.M., et al. (2014). Presence of nanoparticles in wash water from conventional silver and nano-silver textiles. ACS nano, 8(7), 7208-7219.

4. Singh, V., et al. (2006). Applications and future of nanotechnology in textiles, in National Cotton Council Beltwide Cotton Conference, January 3, 2006.

5. Windler, L., et al. (2012). Release of titanium dioxide from textiles during washing. Environmental Science \& Technology, 46(15), 8181-8188.

6. Nowack, B., et al. (2012). Potential scenarios for nanomaterial release and subsequent alteration in the environment. Environmental Toxicology and Chemistry, 31(1), 50-59.

7. Gottschalk, F., Nowack, B. (2011). The release of engineered nanomaterials to the environment. Journal of Environmental Monitoring, 13(5), 1145-1155.

8. Klaine, S.J., et al. (2008). Nanomaterials in the environment: behavior, fate, bioavailability, and effects. Environmental Toxicology and Chemistry, 27(9), 1825-1851.

9. Petersen, A., et al. (2007). Nanotechnologies, risk and society.

10. Wiench, K., et al. (2009). Acute and chronic effects of nano-and non-nano-scale $\mathrm{TiO}_{2}$ and $\mathrm{ZnO}$ particles on mobility and reproduction of the freshwater invertebrate Daphnia magna. Chemosphere, 76(10), 1356-1365.

11. Handy, R.D., Shaw, B.J. (2007). Toxic effects of nanoparticles and nanomaterials: implications for public health, risk assessment and the public perception of nanotechnology. Health, Risk \& Society, 9(2), 125-144.

12. Johnson, D.R., et al. (2010). Potential for occupational exposure to engineered carbon-based nanomaterials in environmental laboratory studies. Environmental Health Perspectives, 49-54.

13. OECD Annual Report (2009).

14. Nerlich, B., Clarke, D.D., Ulph, F. (2007). Risks and benefits of nanotechnology: How young adults perceive possible advances in nanomedicine compared with conventional treatments. Health, Risk \& Society, 9(2), 159-171. 
15. Powell, M.C. (2007). New risk or old risk, high risk or no risk? How scientists' standpoints shape their nanotechnology risk frames. Health, Risk \& Society, 9(2), 173-190.

16. Ganzleben, C., et al. (2011). Review of Environmental Legislation for the Regulatory Control of Nanomaterials: Final Report.

17. Duvall, M., Wyatt, A. (2011). Regulation of nanotechnology and nanomaterials at EPA and around the world: Recent developments and context. Washington, DC: Beveridge \& Diamond, PC.

18. Hertel, T.W. (1997). Global trade analysis: modeling and applications. Cambridge university press.

19. Narayanan, G.B., Angel Aguiar, McDougall, R. (2012). Global Trade, Assistance, and Production: The GTAP 8 Data Base. Center for Global Trade Analysis, Purdue University. 